Research Article

\title{
Investigation of Two Mycobacterium abscessus Outbreaks in Quebec Using Whole Genome Sequencing
}

\author{
Michelle Wuzinski, ${ }^{1}$ Hafid Soualhine, ${ }^{1,2}$ Emilie Valliere, ${ }^{3}$ Pierre-Marie Akochy, ${ }^{4}$ \\ Nancy Cloutier, ${ }^{4}$ Aaron Petkau, ${ }^{5}$ Brynn Kaplen, ${ }^{6}$ Maryse Duchesne, ${ }^{7}$ Jasmin Villeneuve, \\ and Meenu K. Sharma $\mathbb{D D}^{1,2}$ \\ ${ }^{1}$ National Reference Centre for Mycobacteriology, National Microbiology Laboratory, Public Health Agency of Canada, Winnipeg, \\ Manitoba, Canada \\ ${ }^{2}$ Department of Medical Microbiology, University of Manitoba, Winnipeg, Manitoba, Canada \\ ${ }^{3}$ Hopital Sainte-Justine, Montréal, Canada \\ ${ }^{4}$ Laboratoire de Santé Publique du Québec, Montréal, Canada \\ ${ }^{5}$ Bacterial Genomics, Bioinformatics, National Microbiology Laboratory, Public Health Agency of Canada, Winnipeg, \\ Manitoba, Canada \\ ${ }^{6}$ Genomics Core Facility, National Microbiology Laboratory, Public Health Agency of Canada, Winnipeg, Manitoba, Canada \\ ${ }^{7}$ Ministere de la Sante et des Services sociaux, Québec, Canada \\ ${ }^{8}$ DRBST, Institut national de sante publique du Québec, Québec, Canada
}

Correspondence should be addressed to Meenu K. Sharma; meenu.sharma@canada.ca

Received 12 August 2020; Revised 25 September 2020; Accepted 28 October 2020; Published 9 November 2020

Academic Editor: Luis Fernandes

Copyright (c) 2020 Michelle Wuzinski et al. This is an open access article distributed under the Creative Commons Attribution License, which permits unrestricted use, distribution, and reproduction in any medium, provided the original work is properly cited.

\begin{abstract}
In recent decades, nontuberculous mycobacteria (NTM) infections are of emerging public health concern and have contributed towards significant clinical and economic burden globally. One such rapid growing mycobacteria, Mycobacterium abscessus, can cause clonal outbreaks, and these bacteria exhibit a highly resistant antimicrobial susceptibility profile. Here, we present an investigation of two small outbreaks of $M$. abscessus: first in a pediatric clinic setting and second in a tattoo parlour from Quebec. Two whole genome sequencing approaches were utilized for genotyping: MAB-MLST, a multilocus sequencing typing scheme containing housekeeping, identification, and antimicrobial resistance genes, and SNVPhyl that uses phylogenetics to determine single nucleotide variations between strains. MAB-MLST results showed that the pediatric outbreak strains had two distinct sequence types, demonstrating that one strain did not belong to the outbreak, while all tattoo outbreak isolates belonged to the same sequence type. SNVPhyl results were similar to MAB-MLST results and showed that the pediatric outbreak strains tightly clustered together with 0-1 SNVs between isolates, a sharp contrast between unrelated strains used as controls. Similar results were seen for tattoo outbreak cases with 3-11 SNVs between isolates. NTM infections can be difficult to identify, and outbreak investigations can be complicated. Thus, WGS tools can be used in public health outbreak investigations as they provide high discriminatory power.
\end{abstract}

\section{Introduction}

Globally, the incidence of nontuberculous mycobacteria (NTMs) including Mycobacterium abscessus infections has been on the rise [1]. NTMs are ubiquitous environmental organisms, and they can be found in air, soil, and water. These mycobacteria can colonize health-care facilities and commercial and public settings and hence can be responsible for numerous small outbreaks [2,3]. Clinical presentation of one such mycobacteria, M. abscessus, can vary depending on site of infection, but the disease presentation often occurs as soft tissue or pulmonary infections, particularly in those with underlying lung disease such as cystic fibrosis or bronchiectasis. An added disadvantage of M. abscessus infection is its 
resistance to a large number of common antimycobacterial antibiotics. It is referred to as one of the most resistant bacteria known, and thus, treatment is challenging [4]. M. abscessus is divided into three subspecies, M. abscessus subsp. abscessus, M. abscessus subsp. bolletii, and M. abscessus subsp. massiliense $[5,6]$ which have different susceptibilities to antibiotics, and thus, differentiation between them is of vital importance for treatment of infection.

M. abscessus outbreaks can also result from invasive procedures such as cosmetic surgery, tattooing, and acupuncture. Soft tissue infections and surgeries can be responsible for M. abscessus infection via contamination of disinfectants, saline or other fluids, and medical tools [7-10]. Investigation of these outbreaks often involve cumbersome and time consuming strategies such as specimen tracking, laboratory quality control record checks, or via tests for identification and genotyping such as PCR amplification of $16 \mathrm{~S}, r p o B$ or hsp65, random amplification of polymorphic DNA (RAPD), Diversilab rep-PCR genotyping, multilocus sequence typing (MLST), or DNA restriction fragment length polymorphism (RFLP) [11-13].

Nowadays, outbreak investigations have been revolutionized by the implementation of whole genome sequencing (WGS). WGS provides discrimination that was not possible before its advent and hence makes it a powerful tool for not only identification and outbreak investigation but also for surveillance and diagnostics. As a surveillance tool, it adds to contact tracing information and provides evidence-based link of transmission of mycobacteria. Single nucleotide variant (SNV) phylogenomic analysis, such as SNVPhyl [14], can be utilized to construct a phylogenetic tree of microbial genome collection, in order to cluster and compare genomes and infer relatedness. On the other hand, MAB-MLST ( $M$. abscessus-multilocus sequencing typing developed from our laboratory) is an expansion of a traditional MLST scheme to include antimicrobial resistance, identification, and housekeeping genes to generate allele profiles, called sequence types (ST) [15]. Here, we present the use of two whole genome sequencing-based analysis tools, SNVPhyl and MAB-MLST, to retrospectively investigate two suspected outbreaks in the province of Quebec. In order to delineate the transmission of these outbreaks, the whole genome sequencing data for presumptively linked cases stemming from a tattoo parlour and another separate outbreak from a pediatric clinic was ran against both tools.

\section{Materials and Methods}

2.1. Study Strains. M. abscessus isolates were obtained from pediatric patients with otitis of the middle ear and from clients of a tattoo parlour presenting with skin infections. The clinical presentation was skin lesions in the form of multiple 1-2 mm pustules or papules at the tattoo site. Clinical specimens for culture were obtained from patients as described in Table 1. Clinical specimens were decontaminated using standard methodology as described by Clinical and Laboratory Standards Institute [16]. Sediments were cultured to Mycobacterial Growth Indicator Tubes (MGIT) (BD Bactec, UK), Lowenstein Jensen medium, and Middlebrook 7H10
TABLE 1: Case information of outbreak isolates.

\begin{tabular}{lcccccc}
\hline Case \# & NRCM \# & Age & Gender & Outbreak & Specimen type & Site \\
\hline A & 1801480 & 3 & F & Pediatric & Pus & Ear \\
B & 1801479 & 6 & M & Pediatric & Aspiration & Ear \\
C & 1801477 & 4 & M & Pediatric & Swab & Ear \\
D & 1801478 & 1 & F & Pediatric & Pus & Ear \\
E & 1801481 & 60 & F & NR $^{*}$ & Sputum & Lung \\
F & 1801485 & 52 & M & NR & Sputum & Lung \\
G & 1801482 & 20 & M & NR & Sputum & Lung \\
H & 1801483 & 23 & F & Tattoo & Biopsy & Skin \\
I & 1801484 & 30 & M & Tattoo & Biopsy & Skin \\
J & 1801486 & 25 & F & Tattoo & Biopsy & Skin \\
K & 2000565 & 23 & F & Tattoo & Biopsy & Skin \\
\hline
\end{tabular}

${ }^{*}$ NR: nonrelated case.

(MK) agar plates and incubated at $37^{\circ} \mathrm{C}$. The blood agar plate was used to check for contamination at both 24 hours and 48 hours. Culture was grown on MK plates for 4-7 days, evaluated for purity, before being used for DNA extraction. Culture identification was performed by Laboratoire de Santé Publique du Québec using 16S and hsp65 DNA sequencing.

Antimicrobial susceptibility testing (AST) was performed using Trek Sensititre broth microdilution method (Sensititre $^{\mathrm{TM}}$; Thermo Fisher Scientific, Cleveland, OH, USA) using the RAPMYCO plates, and minimum inhibitory concentrations were documented for recommended drugs as per manufacturer's instructions. The interpretation criteria were based on CLSI breakpoints [17]. Isolates were shipped to the National Reference Centre for Mycobacteriology for susceptibility testing and whole genome sequencing.

2.2. Genomic DNA Extraction. A loopful of solid growth from MK plate was added to a $2 \mathrm{~mL}$ tube containing sterile water with glass beads and vortexed. This resulting suspension was adjusted to be $10^{7} \mathrm{CFU} / \mathrm{mL}$ by McFarland, and CFUs were confirmed by colony counts on MK agar plates. An aliquot of $1.5 \mathrm{~mL}$ of the cell suspension was centrifuged for 30 minutes at $4000 \times g$. The supernatant was removed, and cell pellets were frozen at $-80^{\circ} \mathrm{C}$ until ready for DNA extraction.

M. abscessus DNA extractions were performed using the Qiagen DNeasy UltraClean Microbial Kit (Hilden, Germany). Cell pellets were resuspended in $300 \mu \mathrm{L}$ of PowerBead Solution, and then, contents were transferred to a PowerBead tube. $50 \mu \mathrm{L}$ of Solution SL was added and bead beated using the Fast-Prep 24 Tissue and Cell Homogenizer (Hyland Scientific, WA) at $6.5 \mathrm{~m} / \mathrm{s}$ for 4 cycles of 45 seconds each. Manufacturer's instructions were followed thereafter except the final elution step was done twice $(50 \mu \mathrm{L} \times 2)$ to bring the final elution volume to $100 \mu \mathrm{L}$. Samples were then stored at $4^{\circ} \mathrm{C}$ until further processing.

All genomic DNA extracts were quantified using the Qubit 3 Fluorometer (Thermofisher Scientific, CA, USA) as per manufacturer instructions. Samples were diluted to $0.5 \mathrm{ng} / \mu \mathrm{L}$, and $4 \mu \mathrm{L}$ of DNA was used in the sequencing reaction. 
2.3. Whole Genome Sequencing. Sequencing was performed in house using the NexteraXT DNA Library Preparation Kit (Illumina, CA, USA) and sequenced on the Illumina MiSeq (Illumina, CA, USA) with the v3 600 cycle kit $(2 \times 300$ bp reads). The sequences have been submitted to GenBank under Bioproject number PRJNA656566. Quality of the sequences was determined using FastQC (http:// bioinformatics.babraham.ac.uk/projects/fastqc) and SMALT (http://sanger.ac.uk/tool/smalt-0) to map reads to a reference genome. Sequences that mapped at least $85 \%$ of the reference strain (M. abscessus ATCC 19977) and had an average base coverage of at least 50x were included for analysis. Sequences were assembled in the Integrated Rapid Infectious Disease Analysis Project (IRIDA), an open source platform for public health genomics, using the Assembly and Annotation pipeline, which uses Shovill/SPAdes (http://github.com/ tseemann/shovill) for genome assembly $[18,19]$.

All sequences were subjected to phylogenetic analysis using the SNVPhyl pipeline in IRIDA with M. abscessus subsp. abscessus ATCC19977 as the reference genome to generate single nucleotide variant (SNV) matrices and phylogenetic tree [14]. Analysis parameters used were minimum SNV coverage of 15 , minimum mean mapping quality score of 30 , and SNV abundance ratio 0.75 . The SNV density filter was disabled to avoid removal of most of the SNVs for the more distantly related genomes. Second, assembled genomes were genotyped as previously described with MAB-MLST [15] which can be run through the Galaxy platform [20]. Identification, antimicrobial susceptibility testing, and MAB-MLST sequence types for the outbreak strains were analyzed (Table S1 and S2). Statistical comparison was conducted between genotyping and phylogenetic analysis, and any discrepancies were identified as previously described [15].

\section{Results}

Sequences that met quality parameters described in methods were included for bioinformatics analysis. Percent mapping of sequences to the reference ( $M$. abscessus ATCC19977) genome ranged from $86.2 \%$ to $95.7 \%$. Average sequence coverage values for all isolates ranged from $90 \mathrm{x}$ to $151 \mathrm{x}$.

3.1. Pediatric Outbreak. Outbreak isolates B, C, and D were identified as M. abscessus subsp. massiliense both by MABMLST (using $h s p 65$, erm(41), and $r p o B$ alleles) and traditional PCR sequencing of $16 \mathrm{~S}$ and $h s p 65$. Isolate $\mathrm{A}$ and a nonrelated sample E were identified as $M$. abscessus subsp. abscessus. A total of 126299 valid positions (of 138733 total identified positions) were used to generate the SNVPhyl phylogeny shown in Figure 1. The number of single nucleotide differences between pediatric outbreak isolates is shown in Table 2. Isolates B, C, and D clustered closely in the phylogenetic tree, belonged to sequence type 100, and had 0-1 SNVs. $\mathrm{A}$ and $\mathrm{E}$ did not cluster with the outbreak isolates and belonged to sequence types 102 and 101, respectively. Four percent of the total identified positions were filtered out due to coverage.
3.2. Tattoo Outbreak. All tattoo isolates were identified as $M$. abscessus subsp. abscessus by MAB-MLST and PCR sequencing of $16 S$ and $h s p 65$. In addition to the four suspected outbreak cases (isolates $\mathrm{H}, \mathrm{I}, \mathrm{J}, \mathrm{K}$ ) thought to have originated from the tattoo parlour, unrelated outliers $F$ and $G$ were included in the analysis. Isolates $\mathrm{H}, \mathrm{I}, \mathrm{J}$, and $\mathrm{K}$ clustered closely and belonged to sequence type 103. Isolates F and G did not cluster with the outbreak isolates and belonged to sequence type 1 and 83 , respectively. The phylogenetic tree presented in Figure 2 was built from 46464 valid positions identified by SNVPhyl (of 50803 total identified positions). Eight percent of total identified positions were filtered out due to coverage or otherwise invalid. As seen in Table 3, the outbreak isolates have between 3 and 11 SNVs between each other in contrast to the outlier which has between 45933 and 45934 SNVs from the outbreak isolates (Table 3). Further, all suspected outbreak cases were assigned the sequence type (ST) as seen in Table S1 and S2. Drug susceptibility profile for isolate $\mathrm{K}$ was susceptible to clarithromycin with MIC of $2 \mu \mathrm{g} / \mathrm{mL}$, while the related isolates were intermediary to clarithromycin with MIC of $4 \mu \mathrm{g} / \mathrm{mL}$ (Table S1).

\section{Discussion}

Laboratory methods used for infectious disease outbreak investigations have changed drastically over the years as WGS becomes more widespread and integrated as an epidemiological tool [21]. Methods of utilizing WGS data with a high discriminatory index that are fast, reliable, and easy to interpret are invaluable in public health systems. This study describes two separate small outbreaks caused by nontuberculous mycobacteria, Mycobacterium abscessus. One outbreak was detected at a pediatric clinic $(n=4)$ and a second originated from a tattoo parlour $(n=4)$. Both were identified by Public Health Laboratory in Quebec and sent to the National Microbiology Laboratory to be investigated through WGS. Two different methods, MAB-MLST and SNVPhyl, were used to investigate these outbreaks. MAB-MLST is a novel MLST-like scheme that authors previously developed, containing 14 genes involved in both housekeeping and antimicrobial resistance, and generates an output of a sequence type ranging from 1 to 116 [15]. SNVPhyl is an in-house developed IRIDA pipeline at the National Microbiology Laboratory that scans genomes for single nucleotide variants (SNVs), validates them, and uses these variants to build a phylogenetic tree [14].

The first outbreak was suspected in a pediatric clinic in Montreal, Quebec, where four children aged 1-6 presented with symptoms of chronic ear infections between October 2017 and January 2018. Children are known to be more susceptible to infections by NTMs. Fomites, biofilms, water sources, and human to human transmission have all been suggested as source of infection [22]. M. abscessus has been found to inhabit municipal water supplies which can be a major issue in health-care facilities [2]. Due to their inherent resistance to many disinfectants, these organisms can survive on medical devices and spread infection and hence result in a health-care setting outbreak. For this outbreak, it was 


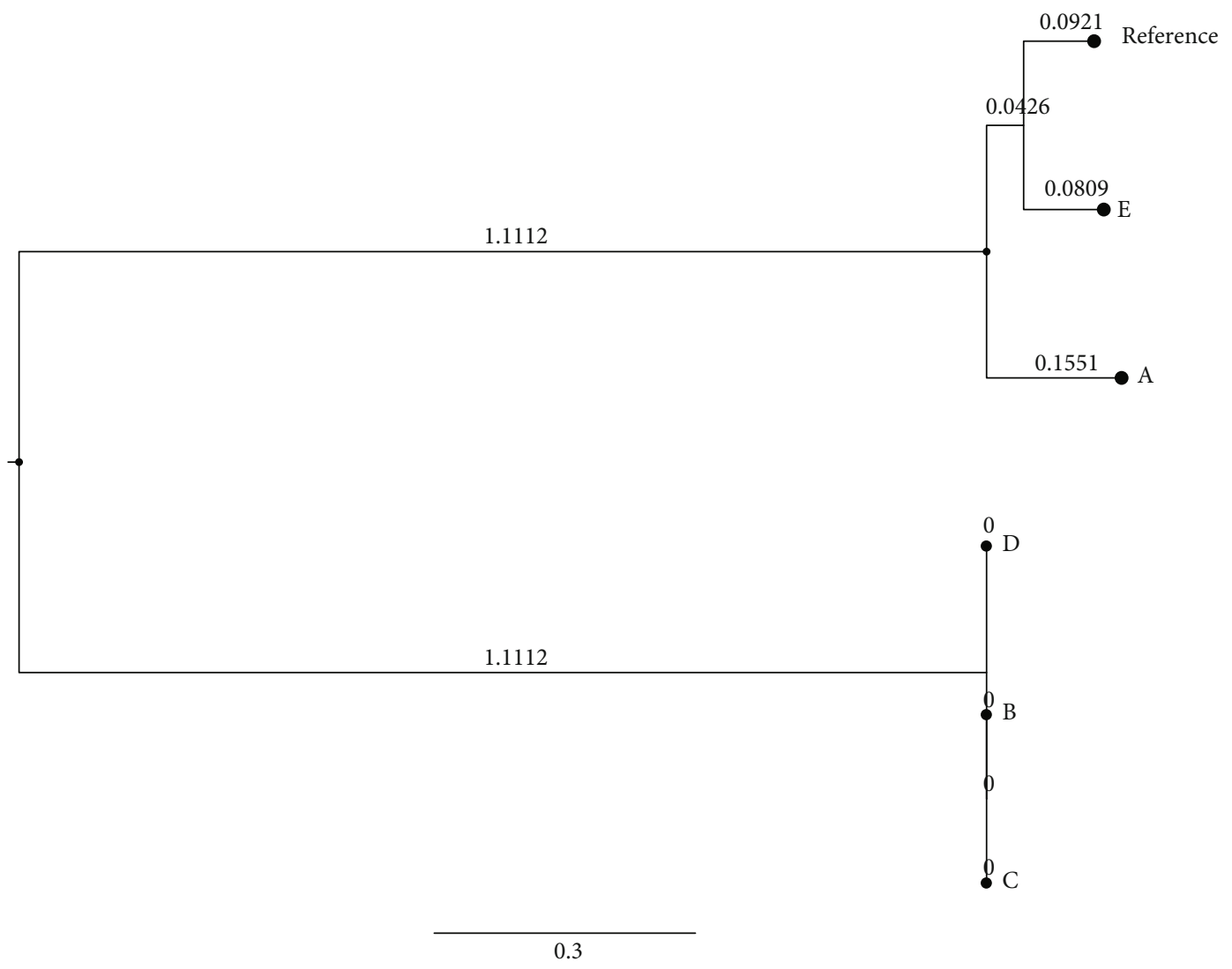

\begin{tabular}{|c|c|c|c|} 
October 2017 & November 2017 & December 2017 & January 2018 \\
\hline A & B & & C, D, E
\end{tabular}

FIgURE 1: Phylogenetic analysis of isolates of suspected outbreak originating from a pediatric clinic. Tree was generated as output from the SNVPhyl pipeline run through IRIDA and visualized in MicroReact. The reference genome refers to ATCC19977. Default SNVPhyl parameters were used with a minimum coverage depth of 15 and mean mapping quality score of 30. Timeline shows date of sample isolation.

TABLE 2: Matrix of single nucleotide differences between pediatric outbreak isolates.

\begin{tabular}{lcccccc}
\hline Strain & $\mathrm{A}$ & $\mathrm{B}$ & $\mathrm{C}$ & $\mathrm{D}$ & $\mathrm{E}$ & Reference \\
\hline $\mathrm{A}$ & 0 & 108610 & 108610 & 108611 & 28650 & 29500 \\
$\mathrm{~B}$ & 108610 & 0 & 0 & 1 & 107181 & 107907 \\
$\mathrm{C}$ & 108610 & 0 & 0 & 1 & 107181 & 107907 \\
$\mathrm{D}$ & 108611 & 1 & 1 & 0 & 107182 & 107908 \\
E & 28650 & 107181 & 107181 & 107182 & 0 & 19259 \\
Reference & 29500 & 107907 & 107907 & 107908 & 19259 & 0 \\
\hline
\end{tabular}

Single nucleotide variations between study isolates as determined by SNVPhyl. Reference is M. abscessus ATCC19977.

hypothesized that a medical device inappropriately decontaminated at a private clinic was the source of infection. An outlier acquired from another hospital (E) was included as a negative control. Of the isolates from the 4 submitted patients, phylogenetic tree and SNV matrix suggest that three isolates belong to the outbreak, and isolate A was acquired from an alternative source. This particular isolate was not only a different sequence type (ST) with different alleles at all 14 loci but belonged to a different subspecies. In contrast, the 3 isolates belonging to the outbreak clustered together and had 0-1 SNVs between them (Table 2; Figure 1). One T to $\mathrm{G}$ mutation at position 3378819 was identified between $\mathrm{D}$ and the other outbreak strains B and C; however, the comparison with M. abscessus ATCC19977 strain suggests that this is not an annotated gene. Due to the near $100 \%$ similarity of the three isolates as well as a single source linked to a medical device from one private clinic, it is concluded that all patients acquired the same strain from the same source at a private clinic.

The second outbreak investigation stemmed from a tattoo parlour. Four patients presented with skin infections with M. abscessus. The time frame of this outbreak was 6 weeks. M. abscessus is a common cause of infections following cosmetic procedures [8]. The SNVs between outbreak strains $\mathrm{H}, \mathrm{I}, \mathrm{J}$, and $\mathrm{K}$ ranged from 3 to 11 . As per Bryant et al., this high degree of genetic similarity suggests that these infections originated from the same source [23]. Strains H, I, J, and K have same ST by MAB-MLST, but susceptibility testing data shows one MIC difference for clarithromycin, making $\mathrm{K}$ strain sensitive to the antibiotic while other 3 strains remain 


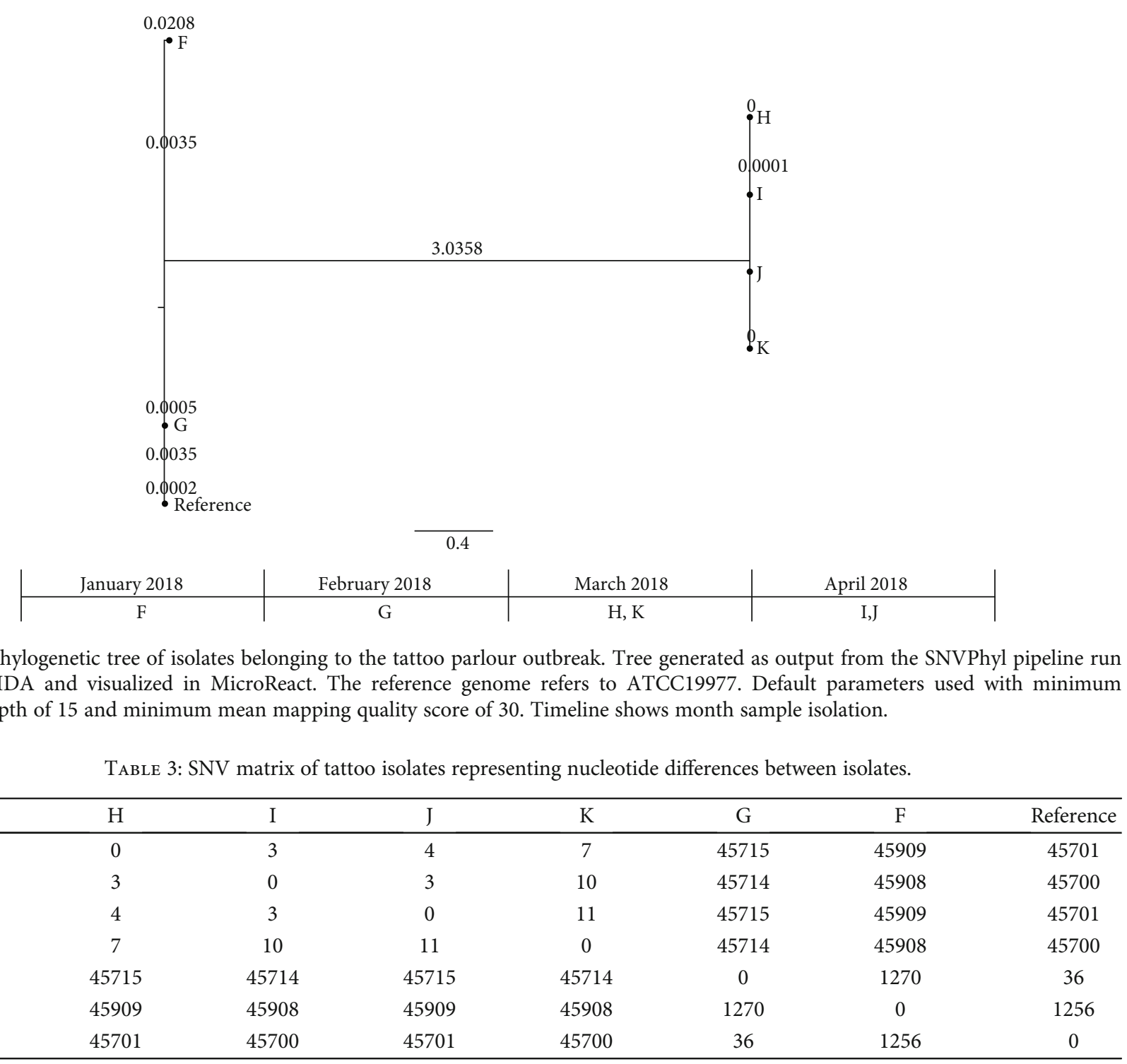

FIgURE 2: Phylogenetic tree of isolates belonging to the tattoo parlour outbreak. Tree generated as output from the SNVPhyl pipeline run through IRIDA and visualized in MicroReact. The reference genome refers to ATCC19977. Default parameters used with minimum coverage depth of 15 and minimum mean mapping quality score of 30 . Timeline shows month sample isolation.

ABLE 3: SNV matrix of tattoo isolates representing nucleotide differences between isolates.

\begin{tabular}{lccccccc}
\hline Strain & H & I & J & K & G & F & Reference \\
\hline H & 0 & 3 & 4 & 7 & 45715 & 45909 & 45701 \\
I & 3 & 0 & 3 & 10 & 45714 & 45908 & 45700 \\
J & 4 & 3 & 0 & 11 & 45715 & 45909 & 45701 \\
K & 7 & 10 & 11 & 0 & 45714 & 45908 & 45700 \\
G & 45715 & 45714 & 45715 & 45714 & 0 & 1270 & 0 \\
F & 45909 & 45908 & 45909 & 45908 & 1270 & 1256 \\
Reference & 45701 & 45700 & 45701 & 45700 & 36 & 0 \\
\hline
\end{tabular}

Single nucleotide variations between study isolates as determined by SNVPhyl. Reference is M. abscessus ATCC19977.

intermediate. This one MIC dilution difference is considered acceptable in a laboratory $[16,17]$. As the clinical data highly suggests the same source for all strains and the number of SNVs between these strains are very low and meet cluster definition by Bryant et al., it can be safely concluded that these strains are related. Bryant et al. used a threshold of less than 25 SNVs to define a cluster; all outbreak strains fall well within this threshold [23]. Of the SNVs unique to the outbreak isolates in Table 3, they were located in 12 chromosomal positions, with the majority found in conserved hypothetical proteins. One of the SNVs found in $\mathrm{H}$ was located in a putative aminoglycoside phosphotransferase (MAB_0951). While M. abscessus is inherently resistant to aminoglycosides through the mechanism of aminoglycoside phosphotransferases, the presence of this SNV showed no effect on amikacin resistance [24]. Multiple SNVs were located different families of transcriptional regulators including the AraC (MAB_1337c), TetR (MAB_1337c and MAB_ 1873c), and the LysR family (MAB_0998). LysR is a diverse family of transcriptional regulators involved in many functions including virulence and metabolism [25]. In mycobacteria, a LysR type regulator, OxyS, was found to regulate the catalase-peroxidase gene and thus is a mechanism for adapting to oxidative stress by regulating katG [26]. This gene is involved in susceptibility to reactive oxygen species and isoniazid in Mycobacterium tuberculosis. However, there was no observable effect on isoniazid antimicrobial susceptibility data in the mutated M. abscessus strain I, as its wild type is intrinsically resistant to isoniazid as well (Table S1).

A recent study utilized WGS to investigate an outbreak of tattoo-associated outbreak of M. abscessus and was able to isolate the source as a bottle of grey wash ink [27]. In our study, the source of infection could not be identified in tattoo outbreak; however, it was hypothesized that tap water bottle was likely a cause since it was routinely used for tattoo ink dilution at the parlour. Drastic infection control measure was put into place including increased hygiene, otoscope disinfection and replacements, medical device disinfection 
protocol revision at the private clinic, disposal material, and reagent changes and disinfection. After a year, no further cases have occurred in either location. Laboratory analysis, time period, SNV, and outbreak origin suggest that these infections were acquired from the same source.

Both WGS-based methods, MAB-MLST and SNVPhyl, showed concordance between the two outbreaks even with a limited number of study isolates. SNVPhyl data provided more discrimination in the tattoo outbreak. The isolate $\mathrm{K}$ had the same sequence type as the other tattoo outbreak isolates (H, I, and J) but had more SNVs identified by SNVPhyl. The number of SNVs between H, I, and J was 3-4 but between $\mathrm{H}, \mathrm{I}$, J, and K was 7-11 SNVs. As per Bryant et al., this difference is less than $25 \mathrm{SNVs}$, hence does not impact clinical outcome, and all 4 isolates belong to the same cluster [23].

The major difference between MAB-MLST and SNVPhyl lies between the output of each respective pipeline. SNVPhyl provides more information on mutated genes, a phylogenetic tree, SNP matrix, or number of mutations present in the entire genome. The significance of each mutation in a specific gene is not easily determined. This data is valuable in research, not easily readable and requires bioinformatics knowledge to decipher this data. MAB-MLST provides direct input on STs as well as which gene profile is present. This gene profile is linked to antibiotic resistance data or to other identification markers since MAB-MLST has housekeeping genes, antimicrobial resistance markers, and markers of identification. This tool is easy to use; output is easy to understand and does not require bioinformatics expertise and hence can be utilized by general technicians in a clinical laboratory. MAB-MLST outputs tabular data with an allele number of each gene and associated ST. This provides an added advantage of directly outputting which genes are mutated and can have implications in easily predicting antimicrobial resistance. In addition, MAB-MLST also has the capability to differentiate the three subspecies by analyzing the $h s p 65, r p o B$, and erm(41) genes of which are all utilized in subspecies identification $[6,28]$. SNVPhyl has higher discriminatory power than MAB-MLST as it uses more genes. It generates multiple file types; the most prominent of which is a phylogenetic tree. While this data is useful in research, knowledge of phylogenetics for data interpretation can pose technical challenges for a diagnostic laboratory worker. SNVPhyl holds an advantage as it provides additional information on relatedness of outbreak strains. It produces a SNV matrix with the number of variants between each strain while MAB-MLST provides sequence type number and lacks SNV information. The applicability of either method is highly dependent on research or diagnostic question.

\section{Conclusion}

Infections caused by nontuberculous mycobacteria, particularly M. abscessus, continue to be a growing health concern. As whole genome sequencing (WGS) becomes commonplace in diagnostic laboratories, its applicability in public health will continue to expand. Both tools utilized WGS data and were used to investigate two suspected outbreaks. MABMLST was able to identify outbreak isolates by assigning the same species and sequence type. However, SNVPhyl was able to go a step further and show that the pediatric outbreak isolates were more closely related than the tattoo isolates by showing single nucleotide differences not identified by MAB-MLST. Utilization of high throughput sequencing for outbreak investigation serves as an invaluable tool for routine mycobacteriology as it provides identification and antimicrobial susceptibility prediction as well as source case identification or genotyping by use of a single laboratory test. Both of these WGS-based methods are useful and can be easily adopted by different laboratories for NTM outbreak investigations.

\section{Data Availability}

The whole genome sequencing data used to in this study have been deposited in the NCBI GenBank under Bioproject number PRJNA656566.

\section{Conflicts of Interest}

The authors declare that they have no conflicts of interest.

\section{Acknowledgments}

The authors would like to thank the NRCM staff for training and support: Alex Gilbert, Alisa McGurran, Catherine Yoshida, Daniel Kein, Darrell Johnstone, Debra Janella, Melissa Rabb, Stephanie Van Driel, and Yvonne Pho. We would also like to thank Bacterial Genomics, Genomics DNA core, Laboratoire de Santé Publique du Québec, and Public Health Quebec for their collaboration.

\section{Supplementary Materials}

Table S1: identification and AST for the outbreak strains. Table S2: full MAB-MLST results for all outbreak cases. (Supplementary Materials)

\section{References}

[1] C. Lin, C. Russell, B. Soll et al., "Increasing prevalence of nontuberculous mycobacteria in respiratory specimens from USaffiliated pacific Island jurisdictions," Emerging Infectious Diseases, vol. 24, no. 3, pp. 485-491, 2018.

[2] R. Thomson, C. Tolson, H. Sidjabat, F. Huygens, and M. Hargreaves, "Mycobacterium abscessus isolated from municipal water - a potential source of human infection," BMC Infectious Diseases, vol. 13, no. 1, p. 241, 2013.

[3] R. J. Wallace Jr., B. A. Brown, and D. E. Griffith, "Nosocomial outbreaks/pseudo outbreaks caused by nontuberculous mycobacteria," Annual Review of Microbiology, vol. 52, no. 1, pp. 453-490, 1998

[4] R. Nessar, E. Cambau, J. M. Reyrat, A. Murray, and B. Gicquel, "Mycobacterium abscessus: a new antibiotic nightmare," The Journal of Antimicrobial Chemotherapy, vol. 67, no. 4, pp. 810-818, 2012.

[5] S. C. Leao, E. Tortoli, J. P. Euzéby, and M. J. Garcia, "Proposal that Mycobacterium massiliense and Mycobacterium bolletii be united and reclassified as Mycobacterium abscessus subsp. bolletii comb. nov., designation of Mycobacterium abscessus 
subsp. abscessus subsp. nov. and emended description of Mycobacterium abscessus," International Journal of Systematic and Evolutionary Microbiology, vol. 61, no. 9, pp. 23112313, 2011.

[6] E. Tortoli, T. A. Kohl, B. A. Brown-Elliott et al., "Emended description of Mycobacterium abscessus, Mycobacterium abscessus subsp. abscessus and Mycobacterium abscessus subsp. bolletii and designation of Mycobacterium abscessus subsp. massiliense comb. nov," International Journal of Systematic and Evolutionary Microbiology, vol. 66, no. 11, pp. 4471-4479, 2016.

[7] M.-R. Lee, W.-H. Sheng, C.-C. Hung, C.-J. Yu, L.-N. Lee, and P.-R. Hsueh, "Mycobacterium abscessus complex infections in humans," Emerging Infectious Diseases, vol. 21, no. 9, pp. 1638-1646, 2015.

[8] E. Y. Furuya, A. Paez, A. Srinivasan et al., "Outbreak of Mycobacterium abscessus wound infections among "Lipotourists" from the United States who underwent abdominoplasty in the Dominican Republic," Clinical Infectious Diseases, vol. 46, no. 8, pp. 1181-1188, 2008.

[9] R. R. Falsey, M. H. Kinzer, S. Hurst et al., "Cutaneous inoculation of nontuberculous mycobacteria during professional tattooing: a case series and epidemiologic study," Clinical Infectious Diseases, vol. 57, no. 6, pp. e143-e147, 2013.

[10] J. Y. Song, J. W. Sohn, H. W. Jeong, H. J. Cheong, W. J. Kim, and M. J. Kim, "An outbreak of post-acupuncture cutaneous infection due to Mycobacterium abscessus," BMC Infectious Diseases, vol. 6, no. 1, p. 6, 2006.

[11] S. C. Leao, E. Tortoli, C. Viana-Niero et al., "Characterization of mycobacteria from a major Brazilian outbreak suggests that revision of the taxonomic status of members of the Mycobacterium chelonae-M. abscessus group is needed," Journal of Clinical Microbiology, vol. 47, no. 9, pp. 2691-2698, 2009.

[12] J. P. N. Singh, R. Verma, and P. Chaudhuri, "Random amplified polymorphic DNA (RAPD) analysis of Mycobacterium tuberculosis strains in India," Journal of Veterinary Science, vol. 7, no. 2, pp. 181-187, 2006.

[13] T. Jagielski, J. van Ingen, N. Rastogi, J. Dziadek, P. K. Mazur, and J. Bielecki, "Current methods in the molecular typing ofMycobacterium tuberculosisand other mycobacteria," BioMed Research International, vol. 2014, Article ID 645802, 21 pages, 2014.

[14] A. Petkau, P. Mabon, C. Sieffert et al., "SNVPhyl: a single nucleotide variant phylogenomics pipeline for microbial genomic epidemiology," Microbial Genomics, vol. 3, no. 6, article e000116, 2017.

[15] M. Wuzinski, A. K. Bak, A. Petkau, W. H. B. Demczuk, H. Soualhine, and M. K. Sharma, "A multilocus sequence typing scheme for Mycobacterium abscessus complex (MAB-multilocus sequence typing) using whole-genome sequencing data," International Journal of Mycobacteriology, vol. 8, no. 3, pp. 273-280, 2019.

[16] Clinical and Laboratory Standards Institute, Susceptibility testing of mycobacteria, Nocardia spp., and other aerobic actinomycetes, Clinical and Laboratory Standards Institute, Wayne, PA, USA, 3rd edition, 2018, CLSI standard document M24.

[17] Clinical and Laboratory Standards Institute, Performance standards for susceptibility testing of mycobacteria, Nocardia spp., and other aerobic actinomycetes, Clinical and Laboratory Standards Institute, Wayne, PA, USA, 1st edition, 2018, CLSI document M62.
[18] T. C. Matthews, F. R. Bristow, E. J. Griffiths et al., "The integrated rapid infectious disease analysis (IRIDA) platform," bioRxiv, no. article 381830, 2018.

[19] A. Bankevich, S. Nurk, D. Antipov et al., "SPAdes: a new genome assembly algorithm and its applications to single-cell sequencing," Journal of Computational Biology, vol. 19, no. 5, pp. 455-477, 2012.

[20] E. Afgan, D. Baker, M. van den Beek et al., "The Galaxy platform for accessible, reproducible and collaborative biomedical analyses: 2016 update," Nucleic Acids Research, vol. 44, no. W1, pp. W3-W10, 2016.

[21] P. J. Stapleton, A. Eshaghi, C. Y. Seo et al., "Evaluating the use of whole genome sequencing for the investigation of a large mumps outbreak in Ontario, Canada," Scientific Reports, vol. 9, no. 1, article 12615, 2019.

[22] M. D. Johansen, J.-L. Herrmann, and L. Kremer, "Non-tuberculous mycobacteria and the rise of Mycobacterium abscessus," Nature Reviews. Microbiology, vol. 18, no. 7, pp. 392407, 2020.

[23] J. M. Bryant, D. M. Grogono, D. Greaves et al., "Wholegenome sequencing to identify transmission of Mycobacterium abscessus between patients with cystic fibrosis: a retrospective cohort study," Lancet, vol. 381, no. 9877, pp. 15511560, 2013.

[24] A. Rominski, P. Selchow, K. Becker, J. K. Brülle, M. Dal Molin, and P. Sander, "Elucidation of Mycobacterium abscessus aminoglycoside and capreomycin resistance by targeted deletion of three putative resistance genes," The Journal of Antimicrobial Chemotherapy, vol. 72, no. 8, pp. 2191-2200, 2017.

[25] S. E. Maddocks and P. C. F. Oyston, "Structure and function of the LysR-type transcriptional regulator (LTTR) family proteins," Microbiology, vol. 154, no. 12, pp. 3609-3623, 2008.

[26] Y. Li and Z.-G. He, "The mycobacterial LysR-type regulator OxyS responds to oxidative stress and negatively regulates expression of the catalase-peroxidase gene," PLoS One, vol. 7, no. 1, article e30186, 2012.

[27] I. Griffin, A. Schmitz, C. Oliver et al., "Outbreak of tattooassociated nontuberculous mycobacterial skin infections," Clinical Infectious Diseases, vol. 69, no. 6, pp. 949-955, 2018.

[28] H. Ringuet, C. Akoua-Koffi, S. Honore et al., "hsp65 sequencing for identification of rapidly growing mycobacteria," Journal of Clinical Microbiology, vol. 37, no. 3, pp. 852-857, 1999. 